\title{
Development and Application of New Dry Barrier Powder Refractory for Aluminum Electrolysis Cell
}

\author{
Changlin $\mathrm{Li}^{12^{2 *}}$, Yanfang Wang ${ }^{1,2}$, Weiguo Bai ${ }^{1,2}$,Yunfeng Zhou ${ }^{1,2}$, Lifen Luo ${ }^{1,2}$, Qingguo Jiao ${ }^{1,2}$, \\ Dengpeng Chai ${ }^{1,2}$ \\ ${ }^{I}$ Zhengzhou nonferrous metals Research Institute Ltd of Chalco Zhengzhou 450041, China. \\ ${ }^{2}$ China National Engineering and Technology Research Centre for Aluminium, Zhengzhou 450041,China.
}

*Corresponding Author: Changlin Li, Zhengzhou nonferrous metals Research Institute Ltd of Chalco, Zhengzhou 450041, China

\begin{abstract}
In order to ensure the quality of barrier powder refractory, avoid losses caused by using poor quality barrier powder refractory, it is imperative to develop high quality barrier powder refractory. A new dry barrier powder refractory with reacting thickness less than $7 \mathrm{~mm}$ in $960^{\circ} \mathrm{C} \times 96 \mathrm{~h}$ test was developed by optimizing the formula. In industrial test, after new dry barrier powder refractory used, electrolytic cell bottom outside surface temperature decreased $37^{\circ} \mathrm{C}$ cell bottom heat loss decreased $18 \mathrm{mV}$, cell bottom kept clean and cell maintained stable in long term operation, cell voltage reduced $25 \mathrm{mV}$, current efficiency increased $0.2 \%, D C$ power consumption decreased $109 \mathrm{kWh} / \mathrm{t}-\mathrm{Al}$, a good energy saving effect was obtained.
\end{abstract}

Keywords: Aluminum electrolysis ; dry barrier powder refractory ; development ; application.

\section{INTRODUCTION}

At present, aluminum is produced by cryolite alumina molten salt electrolysis, and its main equipment is aluminum electrolysis cell. The inner village of the aluminum reduction cell is composed of carbon blocks, refractory bricks and insulation materials. In the long term operation of aluminum reduction cells, cathode refractories are gradually destroyed and lost their protective effect on the underlying insulation materials due to the penetration of electrolyte and steam, resulting in the deterioration of the thermal insulation performance of the cells and the production indicators until the cells are forced to shut down. Therefore, it is the goal to find the furnace building materials which can resist electrolyte penetration. The development and application of dry barrier powder refractory is a breakthrough technological progress in solving electrolyte permeation [1].

Dry barrier powder refractory is an amorphous material, which is made up of different kinds of refractory particles in a certain size ratio. It has both thermal resistance and anti-seepage functions of electrolyte. Dry barrier powder refractory is laid on the insulation layer, and the cathode carbon block can be directly placed on it after compaction, replacing the refractory brick layer and the alumina layer. Dry barrier powder refractory is laid as a whole, without crevices between refractory bricks, which can prevent significant leakage at a certain location, and can partly absorb the cathode expansion power in the vertical direction, thus slowing down the cathode arching up. In addition, the thermal insulation performance of dry barrier powder refractory is better than that of refractory brick, which can ensure the stability of the thermal balance of the cell. Dry barrier powder refractory has good chemical activity, and it can react with the electrolyte penetrated to form dense material layer, which prevents the electrolyte from continuing to leak downward. This is the most fundamental reason for the wide application of dry barrier powder refractory. The technology was rapidly promoted internationally in the early 1990s. In China, China Great Wall Aluminum Corporation first carried out industrial test in 1995. After several years of application, good results have been achieved and considerable economic benefits have been achieved [2].

In recent years, the requirement of refractory materials for large-scale electrolytic cells has been raised. However, some enterprises do not have enough knowledge on functions of dry barrier powder 
refractory and their strict requirements when choosing. Electrolyte begins to penetrate into refractory materials after the start-up of electrolytic cells built with inappropriate dry barrier powder refractory. Thermal performance of electrolytic cells is deteriorating, cell conditions are deteriorating, and electrolytic cell indicators are deteriorating, which increases the operation cost of electrolytic cells. In order to ensure the quality of dry barrier powder refractory and avoid the loss caused by the quality of dry barrier powder refractory to electrolysis enterprises, it is imperative to develop dry barrier powder refractory with high quality and adaptability.

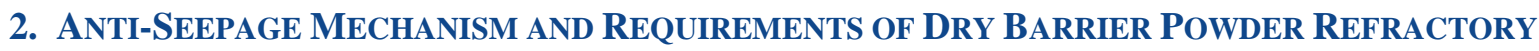

The anti-seepage principle is that the dry barrier powder refractory can react with the electrolyte permeated through the cathode carbon block to form dense layer of glass nepheline or albite, which prevents the continuous penetration of liquid electrolyte and steam and protects the insulation layer. The main reactions are as follows:

$6 \mathrm{NaF}+2 \mathrm{Al}_{2} \mathrm{O}_{3}+9 \mathrm{SiO}_{2}=\mathrm{Na}_{3} \mathrm{AlF}_{6}+3 \mathrm{NaAlSi}_{3} \mathrm{O}_{8}$

The main chemical component of dry barrier powder refractory is $\mathrm{Al}_{2} \mathrm{O}_{3}$ and $\mathrm{SiO}_{2}$. It also contains some other oxides, such as $\mathrm{CaO}, \mathrm{Fe}_{2} \mathrm{O}_{3}, \mathrm{MgO}$, and $\mathrm{TiO}_{2}$. The mass fraction of $\mathrm{Al}_{2} \mathrm{O}_{3}$ and $\mathrm{SiO}_{2}$ is above $80 \%$. According to the current understanding of dry barrier powder refractory research and the needs of field production, high quality dry barrier powder refractory should have the following characteristics $[3,4]$ :

1) Dry barrier powder refractory should have certain chemical reactivity and react quickly with leaking electrolyte to form a barrier layer.

2) When chemical component in a certain range, the higher the vibration density, the better the antiseepage effect of the dry barrier powder refractory.

3) Increasing the melting point of the barrier layer formed by the reaction of dry barrier powder refractory with electrolyte can reduce the consumption of dry barrier powder refractory.

4) Dry barrier powder refractory should be easy to jolt ramming, and the dry barrier powder refractory after vibrating should have considerable bearing capacity.

5) The dry barrier powder refractory does not react with other refractories or insulation materials contacted with the cell lining.

6) Dry barrier powder refractory should not be agglomerated as far as possible under service conditions to increase the difficulty of cell overhauling.

\section{RESEARCH AND DEVELOPMENT OF MATERIALS}

On the basis of previous studies $[4,5]$, the formulation of dry barrier powder refractory was further optimized. The main optimization measures were as follows:

\subsection{Optimizing Component}

The main component of dry barrier powder refractory is $\mathrm{Al}_{2} \mathrm{O}_{3}$ and $\mathrm{SiO}_{2}$. YS/T456-2014 stipulates that the content of $\mathrm{Al}_{2} \mathrm{O}_{3}$ and $\mathrm{SiO}_{2}$ is more than $80 \%$ (wt) and that the content of $\mathrm{SiO}_{2}$ is in the range of $50 \%-60 \%$ (wt). The commonly used component of dry barrier powder refractory is as shown in Table 1[1]. The component of dry barrier powder refractory has a great influence on its physical properties. From the phase diagram of $\mathrm{Al}_{2} \mathrm{O}_{3}-\mathrm{SiO}_{2}$ binary system, it can be seen that the lowest liquid temperature of the two components in the range of 50\%-60\% (wt) is $1546^{\circ} \mathrm{C}$. In the phase diagram of $\mathrm{Al}_{2} \mathrm{O}_{3}-\mathrm{SiO}_{2}$ containing $\mathrm{CaO}, \mathrm{FeO}$ and other oxides, the liquid temperature drops rapidly [6], which indicates that in order to ensure the refractoriness of the two components, it is necessary to limit the impurity content in refractory. In order to ensure the refractoriness of the dry barrier powder refractory, in addition to adding necessary additives, the content of $\mathrm{Al}_{2} \mathrm{O}_{3}$ and $\mathrm{SiO}_{2}$ in the new dry barrier powder refractory was required to be higher than $87 \%$. Improving the refractoriness of dry barrier powder refractory can reduce or even avoid sintering of dry barrier powder refractory particles at high temperature, ensure the reactivity of dry barrier powder refractory and electrolyte, and improve the anti-seepage effect.

Table1. Chemical components of common dry barrier powder refractory

\begin{tabular}{|c|c|c|c|c|c|}
\hline component & $\mathrm{SiO}_{2}$ & $\mathrm{Al}_{2} \mathrm{O}_{3}$ & $\mathrm{Fe}_{2} \mathrm{O}_{3}$ & $\mathrm{CaO}$ & $\mathrm{MgO}$ \\
\hline content\% & $50 \sim 60$ & $30 \sim 35$ & $3.0 \sim 4.0$ & $0.2 \sim 0.5$ & $0.2 \sim 0.5$ \\
\hline
\end{tabular}




\subsection{Optimizing Particle Size Composition}

The particle size composition of dry barrier powder refractory has a great influence on its fluidity, tamping density, anti-seepage performance and thermal conductivity.

The proportion of powder in dry barrier powder refractory increases, the tamping density decreases, which is not conducive to improving anti-seepage performance, but the increase of fine material can reduce the thermal conductivity, which is beneficial to the heat preservation of electrolytic cell, at the same time, there is more fine material in dry barrier powder refractory, the fluidity is enhanced, so it is convenient to build furnaces. It is easy to build the furnace while the increase of fine material in dry barrier powder refractory. Considering the above effects, the new dry barrier powder refractory achieved better performance by controlling the proportion of fine materials. After repeated studies, particle size composition was determined as following: $32 \%<$ fine materials (particle size $<0.74 \mathrm{~mm}$ ) $<38 \%$, and the maximum particle size $<5 \mathrm{~mm}$.

The main performances of the optimized dry barrier powder refractory are as follows:

$\mathrm{Al}_{2} \mathrm{O}_{3}+\mathrm{SiO}_{2} \geq 87 \%$ (wt) ;

$1.94 \mathrm{~g} / \mathrm{cm}^{3}<$ tamping density $<2.0 \mathrm{~g} / \mathrm{cm}^{3}$;

The thickness of reaction layer is less than $7 \mathrm{~mm}$ after $960^{\circ} \mathrm{C} \times 96 \mathrm{~h}$ seepage control test

\section{INDUSTRY APPLICATION}

\subsection{Performance Comparison of Dry Barrier Powder Refractory}

The components and main indexes of new dry barrier powder refractory and dry barrier powder refractory produced by some enterprise were analyzed before industrial test. The components of the two dry barrier powder refractory were shown in Table 2, and the main indexes were shown in Table 3 . The comparison of the anti-seepage performance of the two dry barrier powder refractory was shown in Fig. 1 and Fig. 2.

Table2. Main chemical components of dry barrier powder refractory $1 \%$

\begin{tabular}{|c|c|c|c|c|c|c|}
\hline component & $\mathrm{SiO}_{2}$ & $\mathrm{Al}_{2} \mathrm{O}_{3}$ & $\mathrm{Fe}_{2} \mathrm{O}_{3}$ & $\mathrm{TiO}_{2}$ & $\mathrm{CaO}$ & $\mathrm{MgO}$ \\
\hline $\begin{array}{c}\text { new dry barrier powder } \\
\text { refractory }\end{array}$ & 54.3 & 37.9 & 2.2 & 0.4 & 0.5 & 0.5 \\
\hline $\begin{array}{c}\text { dry barrier powder } \\
\text { refractory produced by } \\
\text { some enterprise }\end{array}$ & 59.7 & 30.3 & 3.9 & 0.8 & 0.4 & 0.3 \\
\hline
\end{tabular}

Table3. Main performance indexes of dry barrier powder refractory

\begin{tabular}{|c|c|c|c|}
\hline index & $\begin{array}{c}\text { amping } \\
\text { density, } \mathrm{g} / \mathrm{cm}^{3}\end{array}$ & $\begin{array}{c}\text { thermal conductivity at } \\
800^{\circ} \mathrm{C}, \mathrm{W} /(\mathrm{m} \cdot \mathrm{K})\end{array}$ & $\begin{array}{c}\text { thickness of reaction layer after } \\
960^{\circ} \mathrm{C} \times 96 \mathrm{~h} \text { seepage control test } \\
\mathrm{mm}\end{array}$ \\
\hline $\begin{array}{c}\text { new dry barrier powder } \\
\text { refractory }\end{array}$ & 1.95 & 0.51 & 6 \\
\hline $\begin{array}{c}\text { dry barrier powder } \\
\text { refractory produced by } \\
\text { some enterprise }\end{array}$ & 1.98 & 0.55 & 15 \\
\hline
\end{tabular}

The anti-seepage performance of the new dry barrier powder refractory was compared with that produced by an enterprise in Fig. 1 and Fig. 2.

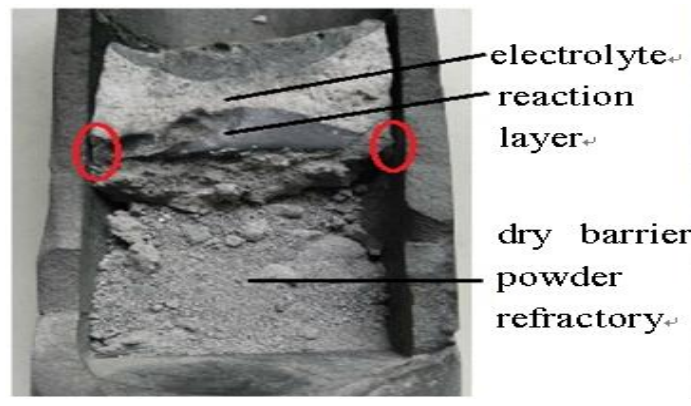

Fig. 1 The result of dry barrier powder refractory produced by some enterprise

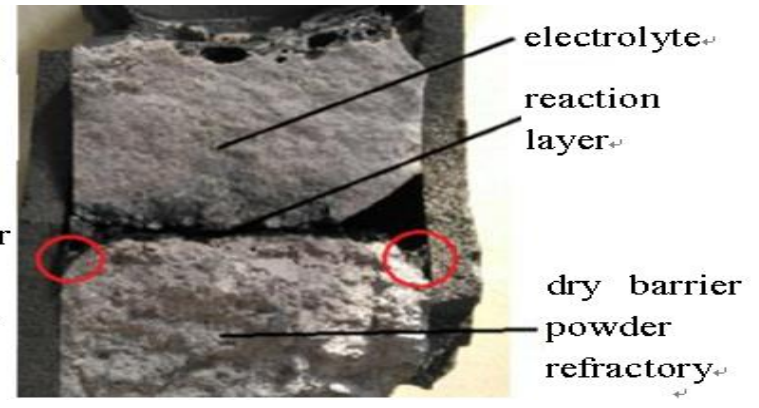

Fig. 2 The result of new dry barrier powder refractory 
Fig. 1 showed the profile of dry barrier powder refractory produced by some enterprise after antiseepage test. Fig. 2 showed the profile of new dry barrier powder refractory after anti-seepage test. It can be seen that both kinds of dry barrier powder refractory had certain anti-seepage effect. A good glass nepheline layer was formed on the surface of the dry barrier powder refractory and the left dry barrier powder refractory was still powdery, which can prevent the liquid electrolyte from continuing to permeate. Comparatively speaking, the anti-seepage effect of the new dry barrier powder refractory was better, the reaction layer was only $6 \mathrm{~mm}$, and the reaction laye thickness of dry barrier powder refractory produced by some enterprise was $15 \mathrm{~mm}$.

\subsection{Comparison of Industrial Application}

In 2016, the new dry barrier powder refractory was applied in 30 cells of $400 \mathrm{kA}$ series in an electrolysis aluminium factory. The design scheme of the bottom lining of the electrolytic cell was $170 \mathrm{~mm}$ thick new dry barrier powder refractory, two layers of light insulating bricks in the bottom insulating layer and $80 \mathrm{~mm}$ calcium silicate board in the bottom layer. After 15 months of operation, the temperature of typical cell bottom surface was as follows. With the same design scheme, the temperature of typical cell bottom surface at the same time built with dry barrier powder refractory from the enterprise was compared as follows.

Table4. Cell bottom surface temperature with different dry barrier powder refractory

\begin{tabular}{|c|c|c|c|c|c|c|}
\hline \multirow{6}{*}{$\begin{array}{c}\text { new dry } \\
\text { barrier powder } \\
\text { refractory }\end{array}$} & position & 1 & 2 & 3 & 4 & 5 \\
\hline & temperature, ${ }^{\circ} \mathrm{C}$ & 75 & 82 & 73 & 74 & 63 \\
\hline & position & 6 & 7 & 8 & 9 & 10 \\
\hline & temperature, ${ }^{\circ} \mathrm{C}$ & 74 & 78 & 72 & 75 & 77 \\
\hline & position & 11 & 12 & 13 & 14 & 15 \\
\hline & temperature, ${ }^{\circ} \mathrm{C}$ & 72 & 67 & 67 & 74 & 69 \\
\hline \multirow{6}{*}{$\begin{array}{l}\text { dry barrier } \\
\text { powder } \\
\text { refractory } \\
\text { produced by } \\
\text { some } \\
\text { enterprise }\end{array}$} & position & 1 & 2 & 3 & 4 & 5 \\
\hline & temperature, ${ }^{\circ} \mathrm{C}$ & 106 & 131 & 103 & 96 & 96 \\
\hline & position & 6 & 7 & 8 & 9 & 10 \\
\hline & temperature, ${ }^{\circ} \mathrm{C}$ & 93 & 98 & 136 & 99 & 111 \\
\hline & position & 11 & 12 & 13 & 14 & 15 \\
\hline & temperature, ${ }^{\circ} \mathrm{C}$ & 104 & 125 & 115 & 111 & 124 \\
\hline
\end{tabular}

It can be seen from the comparison that the temperature of the cell bottom surface decreases greatly and the temperature distribution was uniform after using the new dry barrier powder refractory. The application of the new dry barrier powder refractory laid a foundation for the long-term stable operation of the cell. Within 18 months after the start-up, the bottom of the cell was clean and the operation was stable.

Four cells using dry barrier powder refractory produced by some enterprise and six cells using the new dry barrier powder refractory were randomly selected. The parameters and technical indexes of the cell using two kinds of dry barrier powder refractory at the same time, such as bottom surface temperature, bottom heat dissipation, cell voltage, current efficiency and DC power consumption, were compared. The data are as shown in Table5.

Table5. Effect of dry barrier powder refractory on cell parameters and technical indicators.

\begin{tabular}{|c|c|c|c|c|c|c|}
\hline \multirow{2}{*}{$\begin{array}{c}\text { Material } \\
\text { type }\end{array}$} & $\begin{array}{c}\text { Cell } \\
\text { number }\end{array}$ & $\begin{array}{c}\text { bottom surface } \\
\text { temperature, }{ }^{\circ} \mathrm{C}\end{array}$ & $\begin{array}{c}\text { bottom heat } \\
\text { dissipation, } \mathrm{mV}\end{array}$ & $\begin{array}{c}\text { cell } \\
\text { voltage, V V }\end{array}$ & $\begin{array}{c}\text { current } \\
\text { efficiency, \% }\end{array}$ & $\begin{array}{c}\text { DC power } \\
\text { consumption, } \mathrm{kW} \cdot \mathrm{h} / \mathrm{t}-\end{array}$ \\
$\mathrm{Al}$
\end{tabular}


The average bottom surface temperature of cell using dry barrier powder refractory from the enterprise was $110^{\circ} \mathrm{C}$, and that of cell using new dry barrier powder refractory was $73{ }^{\circ} \mathrm{C}$, which was $37^{\circ} \mathrm{C}$ lower than that of cell using dry barrier powder refractory from the enterprise. The bottom heat dissipation of cell using new dry barrier powder refractory and cell using dry barrier powder refractory from the enterprise were tested and calculated. The average bottom heat dissipation was $133.3 \mathrm{mV}$ and $151.3 \mathrm{mV}$, respectively. Compared with bottom heat dissipation of cell built with dry barrier powder refractory from the enterprise, bottom heat dissipation of cell with new dry barrier powder refractory decreased $18 \mathrm{mV}$. The cell voltage of cell using two kinds of dry barrier powder refractory was $25 \mathrm{mV}$ different. At the same time, for the cell using new dry barrier powder refractory, because of the uniform temperature distribution at the bottom and the stable operation, the statistical current efficiency was $0.2 \%$ higher in 12 months, and the DC power consumption was reduced by $109 \mathrm{kWh} / \mathrm{t}-\mathrm{Al}$.

On August 30 2016, China Nonferrous Metals Industry Association organized a meeting on the evaluation of the results of the project "the new dry barrier powder refractory for aluminium reduction cells" in Zhengzhou. The experts at the meeting finally gave a comprehensive evaluation that the new dry barrier powder refractory was the important direction of scientific and technological development for improving the insulation material of the inner lining of aluminium electrolysis cell, and the overall technology has reached the leading level in China. It is suggested to speed up the popularization and application in electrolytic aluminium industry.

\section{CONCLUSION}

By further optimizing the formula of the dry barrier powder refractory, the anti-seepage and heat preservation effect of the new dry barrier powder refractory was better, and the thickness of the reaction layer was less than $7 \mathrm{~mm}$ after the anti-seepage test at $960^{\circ} \mathrm{C} \times 96 \mathrm{~h}$. The industrial application of the new dry barrier powder refractory showed that the bottom surface temperature of the cell using the new dry barrier powder refractory decreased by $37^{\circ} \mathrm{C}$ compared that of usng dry barrier powder refractory from some enterprise in the same period, and the heat dissipation of the cell bottom decreased by $18 \mathrm{mV}$. During the long-term operation, the bottom of the cell had no precipitation crust and the operation was stable. The cell voltage reduced $25 \mathrm{mV}$, the current efficiency increased $0.2 \%$, and the DC power consumption reduced $109 \mathrm{kWh} / \mathrm{t}-\mathrm{Al}$.

\section{REFERENCES}

[1] Feng Yan. Using Home-made dry preserve heat material at $60 \mathrm{kA}$ soderberg cell with side pins[J]. NONFEROUS MINING AND METALLURGY, 2001, 17(4) : 33-35.

[2] Qun Zhao, Zhuxian Qiu. Application of new material technology in modern large mluminum electrolytic cells [C]. Proceedings of the First China International Conference on Metallurgy and Equipment of Light Metals.

[3] Chen, Zong-an Li, Zhi-qiang Wang, Yu-min Wang, Zhi-qi Zhang, Dao-gao Wu. Study on dry impermeable materials in rare earth molten salt electrolysis [J]. Nonferrous Metals (Extractive Metallurgy), 2014, (2): 43-46.

[4] Shilin Qiu, Dengpeng Cai, Jianhua Xu. Study of anti-leakage chemical materials in aluminum electrolysis [J]. LIGHT METALS, 2000(2): 27-30.

[5] Hong Zhang, Yiren Gan. Development and application of dry Impermeable materials for aluminum electrolysis cell [C]. China Metal Association. Fifth International Conference on Refractories, 1998 (02.

[6] Senlin Xie. Thermodynamic optimization of $\mathrm{CaO}-\mathrm{Al} 2 \mathrm{O} 3, \mathrm{Al} 2 \mathrm{O} 3-\mathrm{SiO} 2$ and $\mathrm{CaO}-\mathrm{SiO} 2$ binary phase diagrams containing C12A7 [D].Ganzhou : Jiangxi University of Science and Technology, 2012.

Citation: Changlin Li, et.al, (2019). Development and Application of New Dry Barrier Powder Refractory for Aluminum Electrolysis Cell. International Journal of Advanced Research in Chemical Science (IJARCS), 6(5), pp.8-12. DOI:http://dx.doi.org/10.20431/2349-0403.0605002.

Copyright: (1) 2019 Authors. This is an open-access article distributed under the terms of the Creative Commons Attribution License, which permits unrestricted use, distribution, and reproduction in any medium, provided the original author and source are credited. 